\title{
Design Cum Performance Equation for a Reactor Type Adsorption Unit
}

\author{
M. A. Islam*, M. S. I. Mozumder, and M. M. R. Khan \\ Department of Chemical Engineering and Polymer Science, Shah Jalal University of \\ Science and Technology, Sylhet-3114, Bangladesh
}

Received 13 January 2009, accepted in revised form 8 July 2009

\begin{abstract}
The conventional method for designing a fixed bed adsorption unit has been discussed. The method is based on the data obtained from an adsorption column test. The characterization of an adsorption system, however, is performed in a laboratory batch experiment. It is shown that the conventional method does not make proper use of the physico-chemical parameters of an adsorption system determined by batch test. Also the method fails to predict the performance of an adsorption unit, if the operating condition differs from that under which the column test has been conducted for design purposes. New design equation has been proposed for both 'Constantly Stirred Tank Reactor (CSTR)' and 'Plug Flow Reactor (PFR)' type adsorption units. The equation predicts the performance of a reactor type adsorption unit under varying operating conditions. The proposed method is based only on the data obtained in batch experiment.
\end{abstract}

Keywords: Adsorption; Unit design; Reactor; Optimal Operation, Dosage; Coefficient of utilization.

(C) 2009 JSR Publications. ISSN: 2070-0237 (Print); 2070-0245 (Online). All rights reserved.

DOI: 10.3329/jsr.v1i3.2592 J. Sci. Res. 1 (3), 450-460 (2009)

\section{Introduction}

Adsorption as a treatment method for liquid and gas is extensively studied both theoretically and experimentally [1-7]. The process is usually applied in chemical and pharmaceutical industries as post-treatment of a stream with a view to improve the quality of the product present in the main stream. As the consciousness of the community rises, the industrial authorities are facing more and more stringent environmental requirements, and trace amount of some component (which is considered to have adverse effect on public health) is to be removed from a fluid stream before being disposed to the environment i.e. the treated stream, in such cases, no longer possesses economical value. For this reason, use of conventional and traditional expensive adsorbents has to be

*Corresponding author: mislam@sust.edu 
abandoned, and search for novel and cost effective adsorbents (which are abundant in nature) has to be initiated. Researchers all over the world have reacted very positively to this demand and hundreds of papers have been published in scientific and technological journals during the past decades, in which the authors report about the adsorption capacity of various types of naturally abundant materials and industrial waste products with respect to some pollutants in a fluid stream. Due to their low cost, these adsorbents need not to be regenerated, and thus appear to be perspective in the treatment of waste fluid before being disposed to the environment. The discovery of the cost-effective adsorbents, which is to be used single time only, dictates the necessity of making a change in the technology as well as in the treatment units. As to the authors' knowledge, so far the researchers have been giving importance to the discovery of cost-effective adsorbents only, leaving the conventional technology and the adsorption units as the only option to be employed. In most studies for characterizing a potential adsorbent, however, the adsorbents are in form of fine particles [1-7]. Small sizes of the particle would provide high resistances and high pressure drop in the operation of an adsorption column [8,9]. Moreover, the adsorbent particles might aggregate resulting in ineffective utilization of the adsorption capacity of the adsorbent. A reactor type adsorption unit would be free of such drawbacks. For designing a reactor type adsorbent, however, requires knowledge about the kinetics of the reaction [10]. Recently Islam et al. [11] has shown that a three-parameter adsorption model would describe the adsorption isotherm as well as adsorption kinetics. These three parameters could form the fundament for analysis of the reaction behavior of finely dispersed adsorbent particles in a reactor type adsorption unit. In the present paper, adsorption unit of the type 'CSTR' and 'PFR' has been proposed as substitute of the conventional 'Fixed Bed Adsorption Unit'. In order to distinguish between the design procedures of the conventional 'Fixed Bed Adsorption Unit' from of the proposed reactor type ones, we have discussed the conventional design procedure and its limitation in describing the performance of the adsorption unit in operating conditions. Then an equation has been derived for the design and also for describing the performance of the proposed CSTR and PFR type adsorption units. The proposed design procedure does not require special laboratory experiments in order to collect data for the design cum performance equation. The results have been illustrated graphically so as to ease the understanding of the new design procedure and the optimal operation of the unit.

\section{Conventional Method for the Design of a Fixed-bed Adsorption Column}

For the design of a fixed bed adsorption column, experiments are conducted on a laboratory scale adsorption column and then the results are scaled up. And these experiments are completely independent of those, which have been conducted for the characterization of adsorbents. Thus, this design method does not make proper use of the results from the previous laboratory studies. The only information being used from earlier experiment is entirely qualitative, which is of the type 'this adsorbent is good for such and such system’. As stated in Geankoplis [9], “A number of theoretical methods have been 
published which predict the mass transfer zone and the concentration profile in the bed. The predicted results are usually inaccurate because of many uncertainties due to flow patterns and correlations to predict diffusion and mass transfer". Thus, for the design of a fixed bed adsorption column, there is no option than to conduct experiments on a laboratory scale adsorption column and collect data for the design. For the purpose, the fluid to be treated is passed through the packed bed at a constant flow rate $u_{0}$ and the concentration of the pollutant $c$ is controlled at the exit of the column. The experimental results are presented as a $c / c_{0}$ vs. $t$ plot as shown in Fig.1, where $c_{0}$ is the pollutant concentration at the entrance of the column and $t$ is the time.

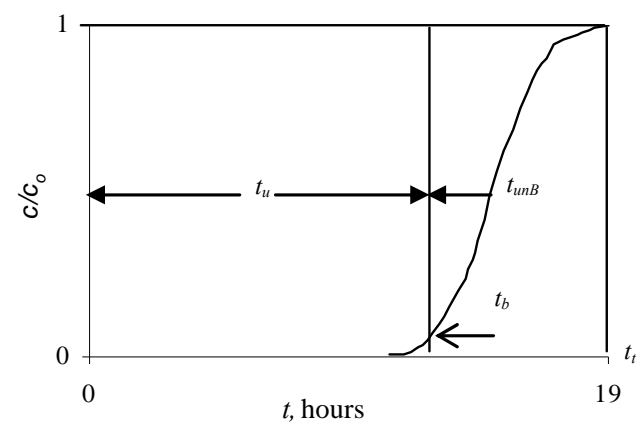

Fig. 1. A typical breakthrough curve for the determining the design parameter of a fixed bed adsorption unit.

In the Fig.1, $t_{b}$ is the time for breakthrough and $t_{t}$ is the time at which the whole adsorbent mass is in equilibrium with the pollutant concentration $c_{0}$. The breakthrough concentration $c_{\mathrm{b}}$ is usually assigned to be equal to (0.01-0.05) $c_{0}$. The time equivalent areas $t_{t}, t_{u}$ and $t_{u n B}$ given by the Eq. (1) represent respectively the total capacity of the bed, the usable capacity, and the unused bed or mass transfer zone.

$$
t_{t}=\int_{0}^{\infty}\left(1-c / c_{0}\right) d t, t_{u}=\int_{0}^{t_{b}}\left(1-c / c_{0}\right) d t, t_{u n \mathrm{~B}}=\int_{t_{b}}^{\infty}\left(1-c / c_{0}\right) d t
$$

The time equivalent areas $t_{t}, t_{u}$ and $t_{u n B}$ are the foundation of the design procedure. In this procedure, it is assumed that the time equivalent area $t_{u n B}$ of the mass transfer zone does not vary with the variation in the concentration at the entrance provided the superficial velocity of the fluid is kept constant. Such assumption merely gives precise results, and for a variation in the superficial velocity $u_{0}$ and the feed concentration $c_{0}$, this design procedure does not provide information about the performance of an already operating adsorption unit.

\section{Design of Reactor Type Adsorption Unit}

In this section, we shall derive the design and performance equation of CSTR and PFR type adsorption unit. Unlike the conventional design procedure, the proposed method is 
completely based on the results obtained from the characterization of the adsorbent by an approach recently developed by Islam et al. [11], and no laboratory scale column test is required. It is considered that the adsorption process could be described by a physicochemical interaction of the type:

$$
A+a c \underset{k_{2}}{\stackrel{k_{1}}{\Leftrightarrow}} a c A, \quad K=\frac{k_{1}}{k_{2}}
$$

where $A, a c \& a c A$ represent respectively the pollutant, active sites and active complexes, and $k_{1} \& k_{2}$ represent the rate constant for adsorption and desorption respectively. These two rate constants together with another Langmuir parameter $q_{\infty}$ characterizing the capacity of the adsorbent form the fundament for the proposed design procedure. The three parameters $k_{1}, k_{2} \& q_{\infty}$ are determined by conventional batch adsorption experiment. For details about the procedure the readers are referred to Islam et al. [11].

\subsection{Design and performance equation for CSTR type adsorption unit}

The mode of operation of a CSTR is presented in Fig. 2 [12,13].

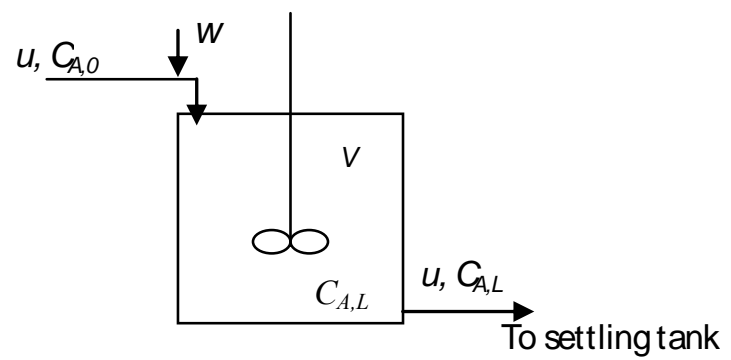

Fig. 2. Mode of operation of a CSTR type adsorption unit.

Let $u\left(\mathrm{~m}^{3} / \mathrm{min}\right)$ is the volumetric flow rate entering and exiting the reactor operating at steady state. Let $C_{A, 0}\left(\mathrm{~kg} / \mathrm{m}^{3}\right)$ be the concentration of the pollutant in the wastewater and $C_{A, L}\left(\mathrm{~kg} / \mathrm{m}^{3}\right)$ be the limit imposed to the industrial authority. Let $w(\mathrm{~kg} / \mathrm{min})$ be the dosage rate of the adsorbent into the reactor. The concept 'dosage rate' should be distinguished from the concept 'dosage' used in Literature [14] describing batch adsorption processes. In a batch experiment, the solution volume is kept constant and the 'dosage' is defined as the amount of adsorbent added per unit volume solution. The reactor system under discussion operates under continuous flow condition and the 'dosage rate' is defined as the amount of adsorbent added to the reactor per unit time. Thus, now if $V\left(\mathrm{~m}^{3}\right)$ be reactor volume (in fact, this is the design parameter, which is to be determined yet), then the following relations express the material balance with respect to the components $A$ (Eq. 3), $a c$ (Eq. 4) and $a c A$ (Eq. 5): 


$$
\begin{gathered}
u C_{A, 0}-u C_{A, L}=\left(k_{1} C_{A, L} C_{a c}-k_{2} C_{a c A}\right) V \\
w q_{\infty}-u C_{a c}=\left(k_{1} C_{A, L} C_{a c}-k_{2} C_{a c A}\right) V \\
-u C_{a c A}=-\left(k_{1} C_{A, L} C_{a c}-k_{2} C_{a c A}\right) V
\end{gathered}
$$

Combining Eqs. (3), (4) and (5), we obtain

$$
C_{a c}=w q_{\infty}-\left(C_{A, 0}-C_{A, L}\right) \& \quad C_{a c A}=C_{A, 0}-C_{A, L}
$$

and

$$
\frac{V}{u}=\frac{C_{A, 0}-C_{A, L}}{k_{1} C_{A, L}\left[w q_{\infty} / u-\left(C_{A, 0}-C_{A, L}\right)\right]-k_{2}\left(C_{A, 0}-C_{A, L}\right)}
$$

Now defining a term 'coefficient of utilization of the capacity of the adsorbent $\eta$ (eta)' as that in Eq. (8), the Eq. (7) may be rearranged as Eq. (9).

$$
\begin{aligned}
& \eta=\frac{u C_{a c A}}{w q_{\infty}}=\frac{u\left(C_{A, 0}-C_{A, L}\right)}{w q_{\infty}} \\
& u / V=k_{I} C_{A, L}(1 / \eta)-\left(k_{I} C_{A, L}+k_{2}\right)
\end{aligned}
$$

The coefficient of utilization $\eta$ (eta)' as defined in Eq. (8) expresses the part of the utilization of the total adsorption capacity of the adsorbent. The $u / V$ vs. $1 / \eta$ plot is a straight line (presented later in Fig. 4). The utilization coefficient $\eta$ would be chosen depending on the cost of the adsorbent, and the design parameter $V$ would be determined from the straight line. In a technological process, the operational parameter $u$ and $C_{A, 0}$ could vary. Then using Eqs. (8) and (9), the dosage rate $w$ would be re-estimated such that the imposed limit $C_{A, L}$ could again be met.

\subsubsection{Maximum achievable $\eta$ and the minimum dosage rate for CSTR}

Eq. (9) shows that as the volume $V$ increases, the utilization coefficient $\eta$ also increases. This is reasonable as with the increase in the reactor volume $V$, the residence/reaction time for the adsorbent increases attaining the maximum value of $\eta=\eta_{\max }$ for $V \rightarrow \infty$. Thus, for $V \rightarrow \infty$ the Eq. (9) reduces to Eq. (10).

$$
\eta_{\max }=\frac{K C_{A, L}}{1+K C_{A, L}}
$$

Combining the Eqs. (8) and 10), for the minimum dosage rate $w_{\min }$, we obtain 


$$
w_{\min }=\frac{u\left(C_{A, 0}-C_{A, L}\right)}{\eta_{\max } q_{\infty}}=\frac{u\left(C_{A, 0}-C_{A, L}\right)}{q_{\infty}}\left(\frac{1+K C_{A, L}}{K C_{A, L}}\right)
$$

As the Eq. (10) shows, the maximum achievable utilization coefficient is limited by the choice of the designed effluent concentration $C_{A, L}$. In fact, the design effluent concentration $C_{A, L}$ is to be chosen as equal to the maximum contamination level (MCL) imposed by the Department of Environment, and as the MCL is lowered, the maximum achievable utilization coefficient also gets lower. Consequently, the minimum dosage rate for the addition of adsorbent in the system has to be chosen higher.

\subsection{Design and performance equation for PFR type adsorption unit}

The mode of operation of a PFR is presented in the Fig. 3 [12,13]. The symbols in the Fig. 3 have the same meaning as those in the Fig. 2. Unlike the CSTR, in a PFR the pollutant concentration decreases gradually along the flow path and hence the material balance will be done in an infinitely small volume $d V$ of the reactor. Let's assume that the reactor has uniform cross-sectional area. Now referring to the Fig. 3, the material balance with respect to the components $A, a c$ and $a c A$ are presented respectively by the Eqs. (12-14).

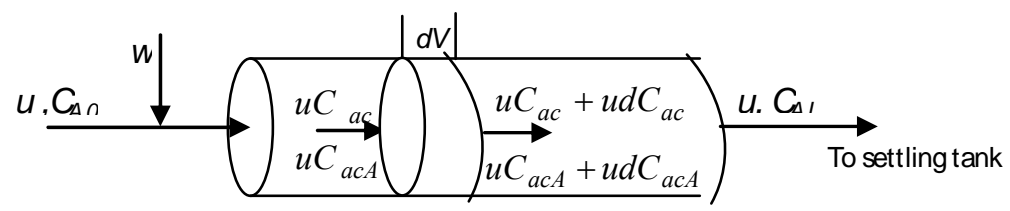

Fig. 3. Mode of operation of a PFR type adsorption unit.

$$
\begin{aligned}
& u C_{A}-\left(u C_{A}+u d C_{A}\right)=\left(k_{1} C_{A} C_{a c}-k_{2} C_{a c A}\right) d V \\
& u C_{a c}-\left(u C_{a c}+u d C_{a c}\right)=\left(k_{1} C_{A} C_{a c}-k_{2} C_{a c A}\right) d V \\
& u C_{a c A}-\left(u C_{a c A}+u d C_{a c A}\right)=-\left(k_{1} C_{A} C_{a c}-k_{2} C_{a c A}\right) d V
\end{aligned}
$$

Combining Eqs. (12-14) we obtain

$$
\begin{aligned}
& C_{a c}=C_{a c, 0}-\left(C_{A, 0}-C_{A}\right), C_{a c A}=C_{A, 0}-C_{A} \text { with } C_{a c, 0}=w q_{\infty} / u \\
& -\frac{V}{u}=\int_{C_{A, 0}}^{C_{A, L}} \frac{d C_{A}}{k_{1}\left(C_{A}^{2}+a C_{A}+b\right)}
\end{aligned}
$$


With,

$$
\begin{aligned}
& a=C_{a c, 0}-C_{A, 0}+1 / K, \quad b=-C_{A, 0} / K \\
& \frac{V}{u}=\frac{1}{k_{l}(\alpha-\beta)} \ln \frac{\left(C_{A, L}-\beta\right)\left(C_{A, 0}-\alpha\right)}{\left(C_{A, L}-\alpha\right)\left(C_{A, 0}-\beta\right)}
\end{aligned}
$$

With

$$
\alpha=\frac{-a+\sqrt{a^{2}-4 b}}{2}, \beta=\frac{-a-\sqrt{a^{2}-4 b}}{2}
$$

For different values of $w, \alpha$ and $\beta$ can be calculated by Eqs. (15), (17) and (19). Then $V$ can be estimated by Eq. (18). It is bit difficult to present the reactor volume $V$ as a simple function of $\eta$. But still the Eqs. (15-19) provide opportunity to generate data for the $u / V$ vs. $1 / \eta$ plot. Fig. 4 shows that beyond a certain value of $\eta$, the plot is linear. Therefore, the relation between $u / V$ and $1 / \eta$ for PFR could be approximated by a straight line (asymptote).

\subsubsection{Maximum achievable $\eta$ and the minimum dosage rate $w_{\min }$ for PFR}

As in CSTR, so in PFR type adsorption unit, the maximum utilization coefficient $\eta_{\max }$ is achieved for possible highest residence time in the unit. Such condition is realizable for $V \rightarrow \infty$. According to Eq. (18), for $V$ to be infinitely large; either $\beta$ must be equal to $C_{A, 0}$ or $\alpha$ must be equal to $C_{A, L}$.

Substituting $\beta=C_{A, 0}$ in Eq.(19), we have

$$
\beta=C_{A, O}=\frac{-a-\sqrt{a^{2}-4 b}}{2}
$$

Eq. (20) could not hold true, as for $a \varepsilon(-\infty, \infty)$, the term in the right hand becomes negative, which would mean that the concentration could be negative. But substituting $\alpha$ $=C_{A, L}$ in Eq. (19), we get

$$
\alpha=C_{\mathrm{A}, \mathrm{L}}=\frac{-a+\sqrt{a^{2}-4 b}}{2}
$$

Now combining Eq. (21) with Eqs. (15, 17), we obtain

$$
\underset{V \rightarrow \infty}{L t} w=w_{\min }=\frac{u\left(C_{A, 0}-C_{A, L}\right)}{q_{\infty}}\left(\frac{1+K C_{A, L}}{K C_{A, L}}\right)
$$

Referring to the definition represented by Eq. (8)

$$
w_{\min }=\frac{u\left(C_{A, 0}-C_{A, L}\right)}{\eta_{\max } q_{\infty}} \Rightarrow \eta_{\max }=\frac{K C_{A, L}}{1+K C_{A, L}}
$$


Now compare Eqs. $(10,11)$ with Eqs. $(22,23)$. As we see, $w_{\min }$ and $\eta_{\max }$ are represented by identical expressions for both CSTR and PFR type adsorption unit.

\subsubsection{Derivation of the Equation of the Asymptote for the design equation of PFR}

The condition $1 / \eta \rightarrow \infty$ could be realized for $w \rightarrow \infty$ i.e. for $a \rightarrow \infty$ (for practical purposes for very high values of $a$ ). Under these conditions, the term $a^{2}$ is much higher than $4 b$. Thus, by some algebraic manipulation of the eq.(19) for $w \rightarrow \infty$, we obtain

$$
\alpha \approx-b / a, \quad \beta \approx-a, \text { and }(\alpha-\beta) \approx a
$$

Substituting Eq.(24) into Eq. (18), we get

$$
\frac{V}{u} \approx \frac{1}{k_{1} a} \ln \frac{C_{A, 0}\left\{1+\left(C_{A, L} / a\right)\right\}\left\{1+\left(b / a C_{A, 0}\right)\right\}}{C_{A, L}\left\{1+\left(b / a C_{A, L}\right)\right\}\left\{1+\left(C_{A, 0} / a\right)\right\}}
$$

Expanding the logarithmic term of the Eq. (25) in a series and neglecting the terms higher than the second order, we obtain

$$
\frac{u}{V}=a_{0} \frac{1}{\eta}-b_{0}
$$

With,

$$
\begin{aligned}
& a_{0}=k_{1} \frac{\left(C_{A, 0}-C_{A, L}\right)}{\ln \left(C_{A, 0} / C_{A, L}\right)} \\
& b_{0}=\frac{k_{2}}{\ln \left(C_{A, 0} / C_{A, L}\right)}\left\{\left(K C_{A, 0}-1\right)-\frac{\left(C_{A, 0}-C_{A, L}\right)\left(K C_{A, L}-1\right)}{C_{A, L} \ln \left(C_{A, 0} / C_{A, L}\right)}\right\}
\end{aligned}
$$

Fig. 4 presents the $u / V$ vs. $1 / \eta$ plot for both the CSTR and the PFR type adsorption unit. The adsorption parameters used in the calculation are as follows: $q_{\infty}=0.04049 \mathrm{~kg} / \mathrm{kg}$, $k_{1}=0.188 \mathrm{~m}^{3} \mathrm{~kg}^{-1} \mathrm{~min}^{-1}$ and $k_{2}=0.00081 \mathrm{~min}^{-1}$ [11]. It is obvious from the Figure the asymptote described by the Eqs. (26-28) could successfully be applied for the design of PFR type adsorption unit. The Fig. 4, also shows that in order to achieve the same concentration limit $C_{A, L}$, the volume of the adsorption unit required for PFR type is much lower than that for CSTR type. This is due to the fact that usually a very low limit of the pollutant concentration is imposed to the industrial authority. Maintenance of such low concentration in the CSTR results in low mass exchange rate, and hence large volume of the CSTR is required to achieve the goal. Prediction of the satisfactory performance of the PFR type adsorption unit makes one to be optimistic that, the pollutant removal might be conducted even in open channel provided the wastewater have sufficient time to be in contact with the adsorbent, and thus special adsorption unit may not be required. 


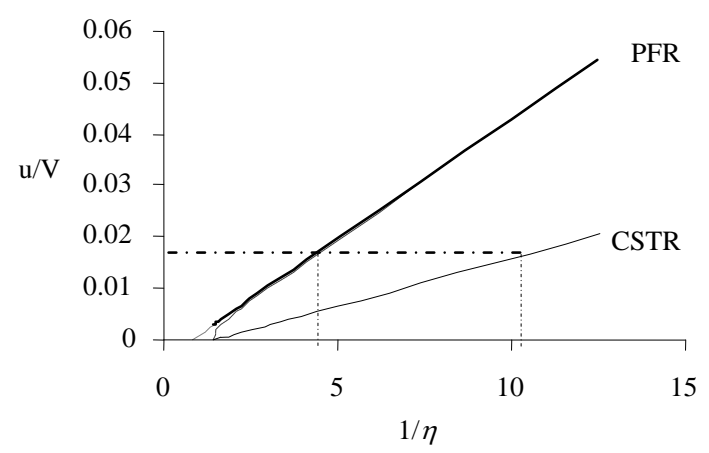

Fig. 4. $u / V$ vs. $1 / \eta$ plot for CSTR and PFR type adsorption unit.

\subsection{Dosage rate calculation in operational conditions}

In the stage of designing, the volume $V$ of a unit is calculated based on an assumed flow rate $u_{0}$ and influent concentration $C_{\mathrm{A}, 0}$. The dosage rate $w_{0}$ is also fixed. In operational condition, however, the flow rate $u$ and influent concentration $C_{\mathrm{A}}$ differ from the design flow rate $u_{0}$ and the design influent concentration $C_{\mathrm{A}, 0}$. In that case, the dosage rate $w$ should be recalculated in order to achieve the same performance as planned during design.

\subsubsection{Dosage rate in an operating CSTR}

Rewriting Eq. (9) for design and operating condition we have

$$
\begin{aligned}
& u_{0} / V=k_{1} C_{A, L}\left(1 / \eta_{0}\right)-\left(k_{1} C_{A, L}+k_{2}\right) \\
& u / V=k_{1} C_{A, L}(1 / \eta)-\left(k_{1} C_{A, L}+k_{2}\right)
\end{aligned}
$$

Combining Eqs. (24) and (25), we find

$$
w=\frac{w_{0}\left(C_{A}-C_{A, L}\right)}{u_{0}\left(C_{A, 0}-C_{A, L}\right)} u-\frac{C_{A}-C_{A, L}}{V k_{1} C_{A, L} q_{\infty}}\left(u_{0}-u\right) u
$$

Now Eq. (31) would give the dosage rate for a CSTR type adsorption unit for which the imposed pollutant limit would be met.

\subsubsection{Dosage rate for PFR}

Re-writing Eq. (26) for design and operating condition we have

$$
\frac{u_{0}}{V}=a_{0} \frac{1}{\eta_{0}}-b_{0}
$$


$\frac{u}{V}=a_{1} \frac{1}{\eta}-b_{1}$

From Eq.(33)

$$
w=\frac{u\left(C_{A}-C_{A, L}\right)}{a_{1} q_{\infty}}\left(\frac{u}{V}+b_{1}\right)
$$

With

$$
\begin{aligned}
& a_{1}=\frac{k_{l}\left(C_{A}-C_{A, L}\right)}{\ln \left(C_{A} / C_{A, L}\right)} \\
& b_{1}=\frac{k_{2}}{\ln \left(C_{A} / C_{A, L}\right)}\left\{\left(K C_{A}-1\right)-\frac{\left(K C_{A, L}-1\right)\left(C_{A}-C_{A, L}\right)}{C_{A, L} \ln \left(C_{A} / C_{A, L}\right)}\right\}
\end{aligned}
$$

The Eq. (34) would give the dosage rate for a PFR type adsorption unit for which the imposed pollutant limit would be met.

\section{Conclusion}

The conventional design procedure for a fixed bed adsorption unit does not make proper use of the adsorption parameters determined in the batch. The method fails to predict the performance of a unit, if the operating conditions differ from those under which the design parameter was estimated. A new design equation has been proposed for the CSTR and PFR type adsorption units. The proposed equation predicts the performance of a reactor type adsorption unit under varying operating conditions. The volume required for PFR type unit is much lower than that for CSTR type unit for the same performances. It is shown that with the lowering of the maximum contamination limit (MCL), the utilization of the capacity of the adsorbents decreases in both CSTR and PFR type reactors, and consequently, for the achievement of a given MCL, the dosage rate is to be chosen higher.

\section{References}

1. V. V. Goud, K. Mohanty, M. S. Rao, and N. S. Jayakumar, Chemical Engineering and Technology, 28 (7), 814 (2005). doi:10.1002/ceat.200500013

2. M. Schäfer, H-J. Schröter, and G. Peschel, Chemical Engineering and Technology 14 (1), 59 (2004). doi:10.1002/ceat.270140109

3. C. A. Eligwe, N. B. Okolue, C. O. Nwambu, and C. I. A. Nwoko, Chemical Engineering and Technology 22 (1), 45 (1999). doi:10.1002/(SICI)1521-4125(199901)22:1<45::AID-CEAT45>3.0.CO;2-D

4. Y. S. Ho and G. McKay, Chem. Eng. J. 70, 115 (1998).

5. G. McKay, H.S Blair, and J. R Gardner, J. Colloid Interface Sci. 95, 108 (1983). doi:10.1016/0021-9797(83)90078-4

6. R. S Juang, R. L Tseng, F. C Wu, and S. H Lee, J. Chem. Technol. Biotechnol. 70, 391 (1997). doi:10.1002/(SICI)1097-4660(199712)70:4<391::AID-JCTB792>3.0.CO;2-V

7. G. McKay, AIChE J. 30, 692 (1984). doi:10.1002/aic.690300434

8. W. L. McCabe, J. C. Smith, and P. Harriott, Unit Operation and Chemical Engineering, Fifth edition (McGraw-Hill, Singapore, 1993). 
9. C. J. Geankoplis, Transport Processes and Unit Operations, Third edition (Prentice-Hall of India, New Delhi, 1999).

10. Metcalf and Eddy, Wastewater Engineering, Third edition (Tata McGraw-Hill Publishing Company Limited, New Delhi, 1999).

11. M. A. Islam, M. R. Khan, and M. S. I. Mozumder, Chemical Engineering and Technology 27, 1095 (2004). doi:10.1002/ceat.200402084

12. O. Levenspiel, Chemical Reaction Engineering, Second edition (Wiley, New York, 1972).

13. H. S. Fogler, Elements of Chemical Reaction Engineering, Second edition (Prentice-Hall of India, New Delhi, 1994).

14. M. S. I. Mozumder, M. M. R. Khan, and M. A. Islam, Asia-Pacific Journal of Chemical Engineering 3 (4), 452 (2008). doi:10.1002/apj.166 